\title{
Adesão pela equipe de enfermagem a lista de verificação cirúrgica: uma revisão
}

\section{narrativa}

\author{
Nursing team's adoption the surgical checklist: a narrative review \\ La adopción del equipo de enfermería la lista de verificación quirúrgica: una revisión narrativa
}

Recebido: 20/01/2021 | Revisado: 23/01/2021 | Aceito: 26/01/2021 | Publicado: 03/02/2021

\author{
Fernanda Soares de Aguir \\ ORCID: https://orcid.org/0000-0001-7211-0186 \\ Universidade Regional Integrada do Alto Uruguai e das Missões, Brasil \\ E-mail: fernandaaguir@hotmail.com \\ Francisco Carlos Pinto Rodrigues \\ ORCID: https://orcid.org/0000-0003-1203-3785 \\ Universidade Regional Integrada do Alto Uruguai e das Missões, Brasil \\ E-mail: francisco@san.uri.br \\ Rosane Teresinha Fontana \\ ORCID: https://orcid.org/0000-0002-0391-9341 \\ Universidade Regional Integrada do Alto Uruguai e das Missões, Brasil \\ E-mail: rfontana@san.uri.br \\ Vivian Lemes Lobo Bittencourt \\ ORCID: https://orcid.org/0000-0003-1488-0611 \\ Universidade Regional Integrada do Alto Uruguai e das Missões, Brasil \\ E-mail: vivillobo@hotmail.com
}

\begin{abstract}
Resumo
A cirurgia segura envolve práticas adotadas para redução dos riscos de eventos adversos e danos que poderão vir a ocorrer no paciente antes, durante ou após a cirurgia. A introdução da lista de verificação para cirúrgica segura, também conhecido como checklist, é um passo para uma nova cultura de segurança na sala operatória. Este estudo tem como objetivo investigar a produção de conhecimento sobre a adesão ao checklist de cirurgia segura. Trata-se de um estudo de revisão bibliográfica sobre a adesão ao uso do checklist no centro cirúrgico. A equipe de enfermagem tem papel essencial na adesão à lista de verificação de cirurgia segura, pois se responsabiliza pela qualificação, comunicação e capacitação profissional, com vistas à melhoria do serviço e a redução de eventos adversos. A adesão ao checklist deve ser aprimorada para contribuir com a redução de eventos adversos e a promoção da segurança do paciente. $\mathrm{O}$ estudo apontou que há várias publicações que demonstram a real efetividade do checklist na literatura, porém se evidenciou que a sua adesão na prática clínica ainda é baixa apontando uma lacuna no conhecimento a ser preenchida pela enfermagem, em especial, as equipes que atuam em centro cirúrgico.
\end{abstract}

Palavras-chave: Segurança do paciente; Centros cirúrgicos; Lista de checagem; Enfermagem perioperatória.

\begin{abstract}
Safe surgery involves practices adopted to reduce the risks of adverse events and damage that may occur in the patient before, during or after surgery. The introduction of the safe surgical checklist, also known as checklist, is a step towards a new safety culture in the operating room. This study aims to investigate the production of knowledge about the access to the checklist of safe surgery. This is a bibliographic review study on the access to the use of the checklist in the operating room. The nursing team plays an essential role in adhering to the safe surgery checklist, as it is responsible for qualification, communication and professional training, with a view to improving the service and reducing adverse events. Checklist adhering should be improved to contribute to the reduction of adverse events and the promotion of patient safety. The study pointed out that there are several publications that demonstrate the real effectiveness of the checklist in the literature, but it was evidenced that its suitability in clinical practice is still low, pointing out a gap in the knowledge to be filled by nursing, especially the teams working in the operating room.
\end{abstract}

Keywords: Patient safety; Surgical centers; Checklist; Perioperative Nursing.

\section{Resumen}

La cirugía segura implica prácticas adoptadas para reducir los riesgos de eventos adversos y daños que pueden ocurrir en el paciente antes, durante o después de la cirugía. La introducción de la lista de verificación quirúrgica segura, también conocida como lista de verificación, es un paso hacia una nueva cultura de seguridad en el quirófano. Este estudio tiene como objetivo investigar la producción de conocimiento sobre el acceso a la lista de verificación de cirugía segura. Se trata de un estudio de revisión bibliográfica sobre el acceso al uso de la lista de verificación en el quirófano. El equipo de enfermería desempeña un papel esencial en la adhesión a la lista de verificación de cirugía 
segura, ya que es responsable de la cualificación, la comunicación y la formación profesional, con el fin de mejorar el servicio y reducir los eventos adversos. Se debe mejorar la verificación de la lista de verificación para contribuir a la reducción de los acontecimientos adversos y a la promoción de la seguridad del paciente. El estudio señaló que hay varias publicaciones que demuestran la eficacia real de la lista de verificación en la literatura, pero se evidó que su idoneidad en la práctica clínica sigue siendo baja, señalando un vacío en el conocimiento que debe llenar la enfermería, especialmente los equipos que trabajan en el quirófano.

Palabras clave: Seguridad del paciente; Centros quirúrgicos; Lista de verificación; Enfermería perioperatoria.

\section{Introdução}

A Organização Mundial da Saúde (OMS) criou em 2004 a World Alliance for Patient Safety (Aliança Mundial para a Segurança do Paciente), com o intuito de dedicar atenção à segurança do paciente, no âmbito mundial. Tem como finalidade coordenar, disseminar e acelerar melhorias para a segurança dos pacientes e seu objetivo principal é diminuir a morbimortalidade de pacientes cirúrgicos e oferecer um instrumento de avaliação uniforme do serviço para a vigilância nacional e internacional (Gama, 2019; Oliveira et al, 2018).

No Brasil, em abril de 2013, instituiu-se o Programa Nacional de Segurança do Paciente, reforçado pela Resolução RDC n 36/2013, da Agência Nacional de Vigilância Sanitária, que estabelece obrigatoriedade para a promoção da segurança do paciente onde há ações voltadas para as cirurgias prescritas em protocolo, que recomendam o uso da lista de verificação cirúrgica, também chamada de checklist de cirurgia segura (Brasil, 2015).

A segurança do paciente envolve os estudos e práticas para a diminuição ou eliminação de riscos desnecessários relacionados à assistência em saúde, com a promoção de ações pelas instituições de saúde e de ensino com finalidade de reduzir a um mínimo aceitável, o risco de prejuízo desnecessário associado ao cuidado em saúde, além de ser um dos seis atributos da qualidade da segurança (Brasil, 2015).

Nesse sentido, o risco do paciente sofrer danos é maior quando os processos organizacionais são planejados ou aplicados de forma inadequada, o Conselho Nacional de Saúde, frisa a importância de dar visibilidade a informações que contribuam para a proteção e prevenção de danos ao paciente, principalmente quando se refere ao processo de inserção de estratégias voltadas à segurança (Brasil, 2016).

A cirurgia segura envolve práticas adotadas para redução do risco de eventos adversos e danos que poderão vir a ocorrer antes, durante ou após as cirurgias, estes por sua vez, são incidentes que resultam em dano ao paciente. A OMS estabeleceu um programa para manter a segurança em cirurgias que consiste na verificação de itens essenciais do processo cirúrgico. Tem como objetivo atender aos cinco certos: paciente, procedimento, lateralidade (lado a ser operado), posicionamento e equipamentos (Instituto Brasileiro de Segurança do Paciente, 2017).

Os problemas relacionados à cirurgia segura ocorrem em grande quantidade e a tensão que esses eventos provocam abalam a confiança da população, dos profissionais e dos sistemas de saúde, por isso acredita-se que a implementação e o uso do checklist é um importante passo para a cultura de segurança não só na sala cirúrgica, como também para uma boa recuperação do paciente na sala de recuperação (Gomes, 2016).

A introdução do checklist é um passo para uma cultura nova de segurança na sala cirúrgica. Realizar a checagem por meio do coordenador, com participação do paciente, familiar e da equipe multiprofissional, é essencial para o procedimento ter sucesso. Quando há trabalho coletivo, os integrantes da equipe passam a se perceber mais do que meros executores de tarefas, e assim contribuem, para uma cirurgia segura e livre de danos (Gomes, 2016; Silva et al, 2019).

Ele torna-se um mecanismo de transformação da prática cirúrgica em hospitais onde é necessária uma política de segurança e, também, um sistema que seja capaz de monitorar e gerenciar os processos organizacionais que efetivamente envolvam o seu uso, sendo este um processo que promove a segurança do paciente. Nesse ínterim, o uso do ckecklist de cirurgia segura tem como objetivo diminuir os atritos e riscos provocados por situações inesperadas e apresentar aos membros 
da equipe antes do procedimento, para garantir a redução de possíveis complicações cirúrgicas e mortalidades nos hospitais (Gomes, 2016).

É conveniente refletir para obter a integração e interação com a equipe, empregando a checagem como meio de comunicação interpessoal, possibilitando a assistência adequada ao paciente, ainda que o relacionamento interpessoal seja o segundo item indicado como agente estressor em profissionais de centro cirúrgico (CC), atrás fica somente a sobrecarga de trabalho (Silva et al, 2016).

Além de promover mudanças no comportamento da equipe, o checklist envolve mudanças no processo de trabalho e colabora na prevenção de eventos adversos. Com a experiência da aplicação, percebeu-se em um estudo de uma revisão integrativa da literatura, realizada por Silva et al no ano de 2016, que apesar da equipe se interessar pelo uso, alguns integrantes não estão preocupados com a mudança de seu comportamento para a realização do mesmo, em contrapartida, quando há o trabalho coletivo, a equipe passa a se perceber a dimensão efetiva do trabalho (Silva et al, 2016).

Admitir que erros acontecem e comunicar o responsável é o primeiro passo para reduzi-los, mas no sistema vigente ide culpa e humilhação, nem tudo o que acontece é notificado, o que impede que outros profissionais aprendam com essas situações. Sendo assim, o processo de trabalho requer uma nova cultura e, por isso, a implantação do checklist em uma sala cirúrgica torna-se essencial para o processo de desenvolvimento da cultura de segurança. Também se orienta que uma única pessoa seja responsável pela aplicação do checklist, tendo plena autoridade e confiança sobre o processo cirúrgico mostrandose apto a interromper o procedimento ou impedir o avanço, se julgar insatisfatório algum dos itens (Souza et al, 2016)

Diante do exposto, o estudo parte do seguinte questionamento: Como a equipe de enfermagem adere ao checklist de cirurgia segura? E, tem como objetivo investigar a produção de conhecimento sobre a adesão da equipe de enfermagem ao checklist de cirurgia segura.

\section{Metodologia}

Trata-se de uma revisão narrativa, segundo Rother (2005) são publicações amplas onde se discute o desenvolvimento de um assunto específico, basicamente uma análise em publicações de livros, artigos e revistas, para o levantamento de dados que já foram produzidos sobre o assunto.

A escolha pela temática ocorreu por se tratar de inquietação dos acadêmicos de enfermagem do sexto semestre de um curso de graduação em enfermagem frente ao conteúdo teórico e prático relacionado à lista de verificação cirúrgica e a elaboração da questão de revisão foi inspirada na estrutura PICo (População, Interesse, Contexto) (Nobre; Bernad; Janete, 2004). A questão norteadora dessa revisão foi: quais as evidências relacionadas à adesão ao checklist de cirurgia segura(I) pela equipe de enfermagem(P) no ambiente cirúrgico hospitalar (Co)?

Como critério de inclusão utilizou-se: artigo relacionado à cirurgia segura e que abordasse o checklist de cirurgia segura; artigos publicados nos últimos cinco anos, entre os anos de 2015 a 2020. A coleta de dados ocorreu entre os meses de agosto e setembro de 2020. Os critérios de exclusão foram: estudos voltados para outros eixos relacionados a segurança de pacientes.

A partir da pergunta de pesquisa e do objetivo, foram definidos os Descritores em Ciências da Saúde: checklist, lista de checagem, segurança do paciente, centros cirúrgicos. Foi utilizado o operador booleano AND entre os descritores de saúde para as buscas. Optou-se por pesquisar nas seguintes bases de dados: Scientific Eletronic Library Online (SciELO), Literatura Latino-Americana em Ciências da Saúde (LILACS) e na Associação Brasileira de Enfermagem de Centro Cirúrgico, Recuperação Anestésica e Centro de Material e Esterilização (SOBECC).

Após leitura exaustiva das publicações na íntegra, incluíram-se as que atenderam a questão de pesquisa. Também se construiu um quadro sinóptico para apresentação dos dados coletados extraídos das publicações. 


\section{Resultados e Discussão}

Do total das 134 publicações decorrentes da exploração inicial, excluindo-se aquelas que não atenderam a questão de pesquisa, permaneceram um total de onze artigos. O ano de publicação variou entre 2018(n=2), 2019(n=5) e 2020(n=4)

Os descritores mais utilizados pelos autores foram "segurança do paciente" e "lista de checagem" nas publicações. Os grupos temáticos que surgiram dos descritores provenientes das publicações foram: fatores dependentes, fatores relacionados e fatores que influenciam na incorporação de protocolos voltados para a cirurgia segura.

A partir da leitura foi organizado um quadro de comparação entre as publicações selecionadas. Neste traz o título do artigo e o ano de publicação, os métodos utilizados para a coleta de dados e por fim os resultados colhidos pelos autores.

Quadro 1 - Caracterização dos títulos e ano de publicação, método e resultados encontrados.

\begin{tabular}{|c|c|c|}
\hline $\begin{array}{l}\text { TITULO DO ARTIGO E } \\
\text { ANO DE PUBLICAÇÃO }\end{array}$ & MÉTODO & RESULTADOS \\
\hline $\begin{array}{l}\text { Adesão do } \text { checklist } \text { cirúrgico à } \\
\text { luz da Cultura de segurança do } \\
\text { paciente. } 2018 \text {. }\end{array}$ & $\begin{array}{l}\text { Revisão integrativa da literatura utilizando as } \\
\text { bases de dados Scientific Eletronic Library } \\
\text { Online (SciELO), Literatura Latino- } \\
\text { Americana em Ciências da Saúde (LILACS), } \\
\text { PubMed e Scopus, no período de janeiro de } \\
2007 \text { a julho de } 2017 \text {. }\end{array}$ & $\begin{array}{l}\text { Dos } 32 \text { estudos que contemplaram o objetivo deste trabalho, } 53,1 \% \\
\text { foram publicados em língua portuguesa e } 40,6 \% \text { no ano de } 2015 \text {. } \\
\text { Entre os temas analisados, destacam-se adesão ao protocolo } \\
(40,6 \%) \text {, registros sobre cirurgia segura }(37,5 \%) \text {, elaboração e } \\
\text { implementação da lista de verificação }(9,4 \%) \text {, percepção dos } \\
\text { profissionais }(9,4 \%) \text { e importância da visita pós-operatória }(3,1 \%) \text {. } \\
\text { Quanto à implementação dos protocolos de cirurgia segura, } 40,6 \% \\
\text { relataram sobre educação permanente e } 21,9 \% \text { sobre } \\
\text { comunicação. }\end{array}$ \\
\hline $\begin{array}{l}\text { Cultura de segurança do } \\
\text { paciente em três hospitais } \\
\text { brasileiros com diferentes tipos } \\
\text { de gestão. } 2018 \text {. }\end{array}$ & $\begin{array}{l}\text { Trata-se de um delineamento observacional } \\
\text { do tipo transversal, com abordagem analítica. } \\
\text { A coleta de dados foi realizada em três } \\
\text { hospitais do Estado do Rio Grande do Norte, } \\
\text { Brasil, com diferentes tipos de gestão, a } \\
\text { saber: federal, estadual e privada. }\end{array}$ & $\begin{array}{l}55,8 \% \text { dos profissionais dos três hospitais classificaram a SP como } \\
\text { boa (nota } 7 \text { ou } 8 \text { ) ou excelente (nota } 9 \text { ou 10) e 22,3\% } \\
\text { classificaram como péssima (nota } 1 \text { ou } 2 \text { ) ou ruim (nota } 3 \text { ou 4). } \\
\text { Em todos os hospitais foi encontrada uma média de } 38 \% \text { para a } \\
\text { proporção de respostas positivas, esta média reflete uma percepção } \\
\text { geral de todas as dimensões da cultura de segurança. }\end{array}$ \\
\hline $\begin{array}{l}\text { Uso do checklist de cirurgia } \\
\text { segura da OMS como } \\
\text { estratégia de redução de } \\
\text { complicações e mortalidade em } \\
\text { cirurgias colorretais: uma } \\
\text { análise de duas realidades, } \\
\text { Brasil X Canadá. } 2019 \text {. }\end{array}$ & $\begin{array}{l}\text { Estudo retrospectivo realizado em um } \\
\text { hospital no Brasil e um hospital no Canadá, } \\
\text { ambos de grande porte, públicos e } \\
\text { universitários. A coleta de dados ocorreu } \\
\text { com revisão de prontuários acerca do } \\
\text { preenchimento do checklist e registros de } \\
\text { complicações até o } 31^{\circ} \text { dia no pós-operatório } \\
\text { de cirurgias colorretais, no período de janeiro } \\
\text { de } 2015 \text { a julho } 2017 \text {. }\end{array}$ & $\begin{array}{l}\text { Foram incluídos } 518 \text { prontuários no Brasil, sendo } 171 \text { (33\%) no } \\
\text { período anterior à implementação do checklist cirúrgico e } 347 \\
(67 \%) \text { posterior à sua implementação; e } 842 \text { prontuários no } \\
\text { Canadá, dos quais } 177 \text { ( } 21 \% \text { ) corresponderam ao período anterior à } \\
\text { implementação do checklist cirúrgico e } 665 \text { ( } 79 \%) \text { posterior a sua } \\
\text { implementação. No que se refere à completude do checklist no } \\
\text { Brasil, dos } 347 \text { checklist, } 222 \text { ( } 64 \%) \text { estavam completos e } 125 \\
\text { (36\%) incompletos. No Canadá, dos } 665 \text { prontuários avaliados } \\
\text { com a presença do checklist, observou-se que } 657 \text { ( } 98,8 \%) \text { estavam } \\
\text { completos e } 8 \text { (1,2\%) incompletos. Quanto aos desfechos, no } \\
\text { hospital do Brasil a taxa de ISC reduziu após a implementação do } \\
\text { checklist de } 17 \% \text { para } 14,4 \% \text {, e os óbitos de } 1,8 \% \text { para } 3,5 \% \text {. No } \\
\text { hospital canadense a taxa de ISC reduziu após a implementação do } \\
\text { checklist de } 27,7 \% \text { para } 25,9 \% \text {, e os óbitos de } 1,7 \% \text { para } 0,9 \% \text {. Em } \\
\text { nenhuma das realidades esses desfechos foram estatisticamente } \\
\text { significativos. Observou-se uma relação direta entre o aumento da } \\
\text { incompletude do checklist e desenvolvimento de ISC no Brasil (p } \\
=0,026) \text {. }\end{array}$ \\
\hline
\end{tabular}




\begin{tabular}{|c|c|c|}
\hline $\begin{array}{l}\text { Cirurgia segura: avaliação da } \\
\text { adesão ao checklist em hospital } \\
\text { de ensino. } 2019 \text {. }\end{array}$ & $\begin{array}{l}\text { Estudo transversal com } 334 \text { pacientes } \\
\text { cirúrgicos ano de } 2015 \text {. Os dados foram } \\
\text { coletados com um instrumento baseado no } \\
\text { checklist padrão da OMS. A amostra foi } \\
\text { descrita por distribuição de frequências. A } \\
\text { prevalência de adesão ao checklist foi } \\
\text { estimada considerando a presença do } \\
\text { instrumento de checagem nos prontuários. } \\
\text { Utilizou análise quantitativa dos resultados. }\end{array}$ & $\begin{array}{l}\text { Verificou-se a existência do checklist em } 90,72 \% \text { dos prontuários. } \\
\text { Nenhuma cirurgia apresentou checklist totalmente preenchido. Em } \\
\text { nenhum instrumento foi encontrado o preenchimento completo dos } \\
\text { três momentos cirúrgicos. A existência do checklist no prontuário } \\
\text { foi associada à classificação da cirurgia quanto à urgência } \\
(\mathrm{OR}=4,3 \text {; IC95\% } \mathrm{p}=1,888,73) \text {. }\end{array}$ \\
\hline $\begin{array}{l}\text { Checklist de cirurgia segura: } \\
\text { adesão ao preenchimento, } \\
\text { inconsistências e desafios. } 2019 \text {. }\end{array}$ & $\begin{array}{l}\text { Trata-se de estudo transversal, documental, } \\
\text { retrospectivo de abordagem quantitativa. A } \\
\text { coleta de dados foi realizada por meio da } \\
\text { revisão retrospectiva de prontuários de uma } \\
\text { amostra de pacientes operados no período de } \\
\text { um ano. Foram incluídos os atendimentos de } \\
\text { pacientes cirúrgicos de todas as } \\
\text { especialidades, com idade de } 18 \text { anos ou } \\
\text { mais, e período de internação igual ou maior } \\
\text { do que } 24 \text { horas. A amostra probabilística foi } \\
\text { de } 423 \text { casos. }\end{array}$ & $\begin{array}{l}\text { O checklist estava presente em } 95 \% \text { dos prontuários. Porém, } \\
\text { apenas } 67,4 \% \text { deles estavam com preenchimento completo. A } \\
\text { presença do checklist no prontuário apresentou associação } \\
\text { significativa com o risco anestésico do paciente. Não houve } \\
\text { diferença no percentual de preenchimento entre os três momentos } \\
\text { do checklist: antes da indução anestésica (sign in), antes da incisão } \\
\text { cirúrgica (time out ou parada cirúrgica) e antes do paciente deixar } \\
\text { a sala de cirurgia (sign out). Também não foram encontradas } \\
\text { diferenças significativas em relação ao percentual de } \\
\text { preenchimento dos itens de responsabilidade do cirurgião. } \\
\text { Considerando o procedimento cirúrgico realizado, foram } \\
\text { encontradas incoerências no item lateralidade. }\end{array}$ \\
\hline $\begin{array}{l}\text { Fatores intervenientes na } \\
\text { implantação do checklist de } \\
\text { cirurgia segura em um hospital } \\
\text { universitário. } 2019 .\end{array}$ & $\begin{array}{l}\text { Estudo qualitativo e análise de conteúdo. } \\
\text { Coleta de dados realizada em } 2017 \text {, por meio } \\
\text { de entrevistas. A população foi composta por } \\
\text { três enfermeiros, quatro residentes de } \\
\text { enfermagem, sete residentes de medicina e } \\
\text { nove técnicos de enfermagem. }\end{array}$ & $\begin{array}{l}\text { Foram agrupados em dois eixos: o primeiro, fatores que dificultam } \\
\text { a implantação do checklist, como modificar a cultura, déficit de } \\
\text { material, resistência da equipe e a burocracia; e, o segundo, fatores } \\
\text { que favorecem a implementação, como o fato de ser hospital } \\
\text { universitário e a qualificação profissional. }\end{array}$ \\
\hline $\begin{array}{l}\text { Lista de verificação de } \\
\text { segurança cirúrgica: benefícios, } \\
\text { facilitadores e barreiras na } \\
\text { perspectiva da enfermagem. } \\
2019 .\end{array}$ & $\begin{array}{l}\text { Estudo transversal, com } 91 \text { enfermeiros em } \\
25 \text { hospitais de dois municípios do Paraná. } \\
\text { Na coleta dos dados, entre } 2015 \text { e } 2016 \text {, } \\
\text { utilizou-se dois instrumentos estruturados. } \\
\text { Para a análise, utilizou-se o teste exato de } \\
\text { Fisher ou Qui-Quadrado. }\end{array}$ & $\begin{array}{l}\text { A implementação do checklist acarretou benefícios para o paciente, } \\
\text { equipe cirúrgica e hospitais. Sobre os facilitadores, os resultados } \\
\text { apresentaram diferença estatisticamente significante entre os } \\
\text { grupos nos itens oferta de educação e aceitação pelos cirurgiões. E, } \\
\text { nas barreiras, para a falta de apoio administrativo e chefias, } \\
\text { ausência do núcleo de segurança do paciente, lista introduzida } \\
\text { abruptamente e ausência de educação. }\end{array}$ \\
\hline $\begin{array}{l}\text { Lista de verificação para } \\
\text { segurança } \quad \text { cirúrgica: } \\
\text { conhecimento e desafios para a } \\
\text { equipe do CC. } 2020 .\end{array}$ & $\begin{array}{l}\text { Pesquisa quantitativa, descritiva, transversal, } \\
\text { realizada em 2017. Fo realizado o } \\
\text { preenchimento de um questionário sobre o } \\
\text { conhecimento e percepções do Protocolo de } \\
\text { Cirurgia Segura por profissionais que atuam } \\
\text { no CC. No total } 72 \text { profissionais de saúde } \\
\text { como médicos, técnicos de enfermagem, } \\
\text { enfermeiros e instrumentadores. A maioria } \\
\text { desses conhecia o Protocolo e objetivos. }\end{array}$ & $\begin{array}{l}\text { O principal desafio para a implantação foi a falta de adesão. } \\
\text { Apontaram planos de condutas que subsidiariam a implantação da } \\
\text { lista na instituição. Os profissionais conhecem a Lista de } \\
\text { Verificação e sabem de sua importância como ferramenta para a } \\
\text { qualidade da assistência durante o perioperatório. Foram listados } \\
\text { os desafios para a implementação prática. }\end{array}$ \\
\hline $\begin{array}{l}\text { Segurança do paciente em CC: } \\
\text { percepção dos profissionais de } \\
\text { enfermagem. } 2020 \text {. }\end{array}$ & $\begin{array}{l}\text { Pesquisa de campo, de caráter exploratório e } \\
\text { descritivo, com abordagem qualitativa. Os } \\
\text { dados foram coletados em um CC por meio } \\
\text { de entrevista gravada norteada por roteiro }\end{array}$ & $\begin{array}{l}\text { As respostas deram origem a seis discursos, que revelaram } \\
\text { preocupação em manter a segurança do paciente por meio de } \\
\text { identificação, comunicação entre equipe multiprofissional e } \\
\text { paciente, prevenção de quedas, ações para a prática segura, }\end{array}$ \\
\hline
\end{tabular}




\begin{tabular}{|c|c|c|}
\hline & $\begin{array}{l}\text { estruturado com quatro questões abertas } \\
\text { fundamentadas no método do discurso do } \\
\text { sujeito coletivo (DSC), com foco na prática } \\
\text { da assistência segura para o paciente no CC. } \\
\text { A amostra foi composta de } 12 \text { profissionais } \\
\text { de enfermagem, sendo um auxiliar e } 11 \\
\text { técnicos. Os dados foram organizados e } \\
\text { analisados segundo método do DSC. }\end{array}$ & comunicação intersetorial e manutenção de equipamentos. \\
\hline $\begin{array}{l}\text { Cirurgia segura: análise da } \\
\text { adesão dos médicos aos } \\
\text { protocolos e seu potencial } \\
\text { impacto na segurança do } \\
\text { paciente. 2020. }\end{array}$ & $\begin{array}{l}\text { Este é um estudo transversal e prospectivo. } \\
\text { Foi desenvolvido um instrumento para } \\
\text { coletar as características sociográficas de } \\
\text { sessenta e oito cirurgiões e residentes, seus } \\
\text { conhecimentos e adesão ao protocolo de } \\
\text { cirurgia segura. Oitenta e dois pacientes } \\
\text { foram avaliados quanto à sua conscientização } \\
\text { sobre o procedimento cirúrgico. O ambiente } \\
\text { operacional também foi avaliado. Estatísticas } \\
\text { descritivas e a razão de chances são } \\
\text { apresentadas. }\end{array}$ & $\begin{array}{l}\text { Os cirurgiões, apesar de seu contato prévio com o protocolo } \\
\text { durante todo o período de graduação, foram mal em conformidade } \\
\text { com ele. Foram identificados eventos adversos como o uso de } \\
\text { equipamentos não calibrados ou a presença de corpos estranhos em } \\
\text { diversos equipamentos, como brocas e canetas cautery. Além } \\
\text { disso, alguns dos eventos adversos foram identificados e } \\
\text { corrigidos, após os pacientes já terem sido anestesiados, mas antes } \\
\text { do início do procedimento cirúrgico. Os pacientes demonstraram } \\
\text { conhecimento sobre a operação que seriam submetidos, mas não } \\
\text { sabiam da duração e não foram apresentados à equipe cirúrgica. }\end{array}$ \\
\hline
\end{tabular}

Fonte: Fernanda Soares de Aguir (2020).

Como podemos observar no quadro acima, os resultados são diversos, porém conseguimos observar que na grande maioria dos artigos a adesão em teoria é muito boa, porém foram encontradas barreiras quando aplicado na prática como por exemplo, falta comunicação multiprofissional, resistência por meio da equipe em aplicar a lista de checagem, muita burocracia e falta de ações educativas por meio dos gestores.

No Brasil, às listas de verificação vêm sendo usadas nos serviços de saúde, porém com alguns desafios, como a conscientização indevida da importância de seu uso pelos profissionais da equipe cirúrgica, com sinais, ainda, de culpabilização e indenizações pelas práticas inseguras. A viabilidade da lista de verificação de segurança cirúrgica vem se apresentando promissora em diversos hospitais brasileiros, embora haja, ainda, baixo engajamento da equipe cirúrgica em sua adesão. O enfermeiro, como coordenador do setor cirúrgico, pode se apropriar dessa ferramenta como forma de mensurar e avaliar a assistência prestada (Oliveira et al, 2018).

A utilização de checklist está sendo executada por diversas instituições de saúde, diante de seu valor e sua cultura organizacional, o que se sucede em um benefício tanto para os profissionais quanto para os pacientes. Pode-se obter vários dados de intervenção mais confiáveis e sistemáticos dos modelos de alto nível de organização que demonstram melhoria na segurança dos serviços de saúde como um todo durante a implementação de checklists voltados para a cirurgia segura décadas atrás (Oliveira et al, 2018).

Para isso, a equipe precisa estar engajada e comprometida individual e coletivamente, de modo a compreender a importância e a necessidade do uso da lista de verificação e, a posteriormente, atribuir ações corretivas por meio de indicadores seguros. A enfermagem responsabiliza-se pelo planejamento de suas ações, pelo dimensionamento de pessoal, preconizado pela Portaria $n^{\circ}$ 543/2017 do Conselho Federal de Enfermagem (COFEN) e pelas capacitações e pelos treinamentos das equipes de trabalho no cuidado seguro (Oliveira et al, 2018).

Todas essas ações visam à melhoria nos padrões de cuidado mediante comunicação interprofissional segura e redução de danos físicos e psicológicos ao paciente nesse ambiente de saúde. Assim, a equipe de enfermagem tem papel essencial na adesão à lista de verificação, pois se responsabiliza pela qualificação, comunicação e capacitação profissional, com vistas à 
melhoria do serviço e à redução de eventos adversos (Oliveira et al, 2018).

\section{Conclusão}

O estudo apontou que há várias publicações que demonstram a real efetividade do checklist na literatura, porém se evidenciou que a sua adesão na prática clínica ainda é baixa apontando uma lacuna no conhecimento a ser preenchida pela enfermagem, em especial, as equipes que atuam em CC. Soma-se a isso o compromisso ético das instituições e organizações a reconfigurarem os seus processos de trabalho para a melhoria na segurança do paciente em todos os eixos preconizados pela OMS.

Esperamos que em trabalho futuros possamos observar uma realidade diferente, tanto na parte teórica como na parte prática, que os autores consigam trazem a tão esperada adesão correta ao uso do checklist em suas publicações, podendo assim, trazer mais segurança tanto para os pacientes como para a equipe multiprofissional envolvida e fazendo com que o cliente saia satisfeito do procedimento.

Por fim, torna-se um desafio à participação efetiva da enfermagem por meio de diálogos entre a equipe, o paciente, os familiares e os gestores do hospital na efetivação do checklist de cirurgia segura. Essas estratégias ampliam a participação da equipe e redobram a atenção ao paciente, além de fortalecem o cuidado de seguro.

\section{Referências}

Andrade, L. E. L., et al. (2018). Cultura de segurança do paciente em três hospitais brasileiros com diferentes tipos de gestão. Ciênc. saúde colet.

Brasil. Ministério da Saúde. (2015). Documento de referência para o Programa Nacional de Segurança do Paciente / Ministério da Saúde; Fundação Oswaldo Cruz; Agência Nacional de Vigilância Sanitária. Brasília: Ministério da Saúde.

Conselho Federal de Enfermagem. (2019). Portaria $n^{\circ}$ 543/2017.

Ferreira, R., Alves et al. (2019). Fatores intervenientes na implantação do checklist de cirurgia segura em um hospital universitário. Enferm. Foco, Brasília, 10(2): 41-46.

Gama, C. S. (2019). Uso do Checklist de cirurgia segura da Organização Mundial da Saúde como estratégia de redução de complicações e mortalidade em cirurgias colorretais: uma análise de duas realidades, Brasil X Canadá. Belo Horizonte.

Gomes, C. D. P. P., et al. (2016). Percepção de uma equipe de enfermagem sobre a utilização do checklist cirúrgico. Rev. SOBECC, São Paulo. JUL./SET.

Instituto brasileiro de segurança do paciente. (2017). Segurança e paciente.

Marquioni, F. S. do N., et. al. (2019). Cirurgia segura: avaliação da adesão ao checklist em hospital de ensino. Rev. SOBECC.

Oliveira, M. C. B. de, et. al. (2018). Adesão do cheklist cirúrgico à luz da cultura de segurança do paciente. Rev. SOBECC.

Ribeiro, L., et al. (2019). Checklist de cirurgia segura: adesão ao preenchimento, inconsistências e desafios. Rev. Col. Bras. Cir. $46(5)$, e20192311.

Rother, E. T. (2007). Revisão sistemática X revisão narrativa. Acta Paulista de Enfermagem, 20(2),. v-vi.

Santos, E. A., et al (2020). Lista de verificação para segurança cirúrgica: conhecimento e desafios para a equipe do centro cirúrgico. Enfermería Actual de Costa Rica. (38), 75-88.

Silva, A. T., et. al. (2016). Assistência de enfermagem e o enfoque da segurança do paciente no cenário brasileiro. Saúde debate [online]. 40(111), 292-301.

Silva, P. H. A., et al. (2020). Safe surgery: analysis of physicians' adherence to protocols, and its potential impact on patient safety. Rev Col Bras Cir. 47: e20202429.

Souza, A. T. G., et al. (2020). Segurança do paciente em centro cirúrgico: percepção dos profissionais de enfermagem. Rev. SOBECC; $25(2)$ : 75-82.

Souza, R. M., et. al. (2016). Aplicabilidade de checklist de cirurgia segura em centros cirúrgicos hospitalares. Rev. SOBECC.

Tostes, M. F. do P., \& Galvao, C. M. (2019). Lista de verificação de segurança cirúrgica: benefícios, facilitadores e barreiras na perspectiva da enfermagem. Rev. Gaúcha Enferm. 40, e20180180. 